\title{
TERAPIA COMPORTAMENTAL CONSTRUTIVA: UMA OUTRA FACE DA CLÍNICA COMPORTAMENTAL
}

\author{
Luc Vandenberghe ${ }^{1}$
}

Resumo: Este artigo apresenta a terapia comportamental construtiva conforme elaborada por Beata Bakker-de Pree. A abordagem, desenvolvida nos Países Baixos, traz uma filosofia do processo terapêutico que é compatível com a de várias terapias comportamentais conhecidas. Acrescenta uma perspectiva valiosa em sua descrição explícita de alguns ingredientes importantes do trabalho clínico, que não estão em destaque nos outros modelos comportamentais, sendo estas a técnica de diferenciação livre e a análise funcional do comportamento saudável do cliente.

Palavras-chave: Terapia comportamental. Behaviorismo. Modelo clínico.

A terapia comportamental: um histórico

A primeira onda de terapias comportamentais ambulatoriais emergiu nos anos 1950 como movimento mundial. Foi fortemente identificada como uma tentativa de substituir a psicoterapia, que era considerada ineficaz e anti-científica (Eysenck, 1952, 1959). Tinha um embasamento predominantemente pavloviano, mas não aderiu a um modelo único de aprendizagem humana (Wolpe, 1976). Eysenck (1959) descreveu a terapia comportamental em termos da eliminação de respostas inadequadas e do condicionamento de respostas adequadas - porém, apenas o primeiro aspecto é que ia dominar a tecnologia clínica. Os métodos clássicos se baseavam, na sua grande maioria, nos princípios de extinção pavloviana. Assim, a terapia comportamental se desenvolveu como um tratamento para eliminar respostas emocionais inadequadas. 
A busca de uma tecnologia para aquisição de novos comportamentos despertou em alguns o interesse pelos métodos da análise aplicada do comportamento, que poderia preencher essa lacuna. De fato, contribuições a partir do modelo de aprendizagem operante para a terapia comportamental não foram escassas, mas sua aceitação no seio do movimento não foi geral, nem durável. Reforçar sorrisos ou afirmações otimistas de um cliente depressivo (Coons, 1972; Krasner, 1962) ou montar um sistema de economia de fichas para ser aplicado em casa como tratamento de uma crise de casal (Stewart, 1969) pareceu indicar, aos olhos dos clínicos pavlovianos, uma visão muito superficial. Os terapeutas comportamentais da primeira onda estavam mais interessados nas respostas emocionais subjacentes ao comportamento depressivo e aos problemas de relacionamento. Concluíram que o paradigma operante tinha uma validade questionável para o tratamento dos problemas que eles enfrentaram no consultório.

Essa rejeição da tecnologia operante pela terapia comportamental clássica é atribuída por Kohlenberg, Bolling, Kanter e Parker (2002) ao uso de procedimentos artificiais. Os analistas do comportamento usaram no consultório reforçamento planejado para ensinar comportamentos que haviam escolhido como sendo adequados, em detrimento do relacionamento genuíno entre terapeuta e cliente. Isto pode ter contribuído para a separação que ocorreu entre a análise aplicada do comportamento, de um lado, e a terapia comportamental tradicional, do outro. Afastada do setting de gabinete, a primeira continuou florescendo em trabalhos com crianças, pessoas portadoras de deficiência mental e pacientes internados com transtornos psiquiátricos graves. A terapia comportamental, por sua vez, continuou se dedicando a transtornos de humor ou de ansiedade, problemas de casal e outras queixas que caracterizam o trabalho no consultório ambulatorial.

O que podemos chamar a segunda onda na clínica comportamental surgiu com o advento dos modelos cognitivos, segundo os quais comportamentos interpessoais e emoções são mediados por estruturas e processos cognitivos. Foi promovida por uma nova geração de clínicos, a qual permaneceu crítica em relação a assunções que não tinham sido investigadas empiricamente, e se pautou no mesmo modelo hipotéticodedutivo usado pela terapia comportamental clássica. Manteve, assim, uma continuidade com o ideal das ciências empíricas que tinha sido defendido pelos neo-pavlovianos. Com suas inovações técnicas, a terapia cognitivo-comportamental transformou o tratamento dos transtornos de humor (Beck, Rush, Shaw, \& Emery, 1979; Ellis \& Grieger, 1977) e chegou a se impor no campo dos transtornos de ansiedade que, durante décadas, havia sido o domínio do modelo pavloviano (Craske \& Rowe, 1997; Rachman, 1998). Enquanto teve um desempenho marcante nas pesquisas de eficácia, tanto autores skinnerianos (e.g. Jacobson, 1997) 
quanto neo-pavlovianos (e.g. Eysenck, 1987) lançaram objeções de natureza paradigmática e epistemológica contra o movimento.

A terceira onda se caracteriza por um retorno ao processo psicoterápico vivencial, no intuito de superar as limitações impostas pela noção, cultivada pelas terapias comportamental clássica e cognitivocomportamental, de que o tratamento se restringe à aplicação de técnicas. Destaca-se também pela adoção de visões de mundo que são diferentes da epistemologia linear tradicional, como o construtivismo social (Neimeyer \& Mahoney, 1995/1997) ou releituras contextualistas do behaviorismo radical (Dougher \& Hayes, 2000; Kohlenberg,Tsai \& Dougher 1993). Análise clínica do comportamento é a denominação coletiva usada para quatro diferentes abordagens pertencentes a essa terceira onda: a psicoterapia analítico-funcional (Kohlenberg \& Tsai, 1991/2001), a terapia de aceitação e compromisso (Hayes, 1987; Hayes, Strosahl, \& Wilson, 1999), a terapia comportamental integrativa de casal (Jacobson \& Christensen, 1996) e a terapia comportamental dialética (Linehan, 1993). As quatro emergiram nos anos 1980 como psicoterapias fundamentadas numa análise do comportamento, que queria se distinguir claramente da análise aplicada do comportamento pela adoção do adjetivo "clínica" em vez de "aplicada".De um ponto de vista histórico, a análise clínica constitui o retorno da filosofia analítico-funcional na prática de consultório através de uma releitura do processo terapêutico em termos contextualistas. A opção de analisar o processo terapêutico e não o comportamento do indivíduo em si torna impossível recorrer ao uso de reforçamento planejado ou a contingências análogas. Essa opção fez com que o rompimento com a análise aplicada do comportamento chegasse a ser total. Restava somente a opção de trabalhar com a vivência genuína e espontânea do cliente e do terapeuta.

O presente artigo descreve uma forma de terapia comportamental que se desenvolveu nas margens da história acima relatada, no seio de uma comunidade geograficamente circunscrita, e aponta para algumas contribuições que a mesma pode fazer para o movimento da terapia comportamental mundial. Finalmente, a compatibilidade dessa terapia com a filosofia da terceira onda é discutida.

\section{A visão construtiva no consultório}

Israel Goldiamond (1974) observou que os comportamentos disfuncionais constituem, junto com os comportamentos adequados de uma mesma pessoa, uma construção única, em que uma parte não pode ser modificada sem afetar o todo. Repertórios que consideramos inadequados, por causarem problemas no cotidiano do paciente, podem ter funções importantes para essa pessoa. De um ponto de vista operante, as funções são responsáveis pela manutenção dos repertórios. Quando 
novos comportamentos, mais adequados, surgem para desempenhar as mesmas funções, os comportamentos inadequados perdem sua utilidade. A implicação mais importante desse raciocínio para a clínica é que o terapeuta comportamental não deve se concentrar na eliminação dos sintomas de depressão ou ansiedade, mas ajudar o paciente a desenvolver estratégias de vida diferentes, mais saudáveis, menos dolorosas, que possam tomar o lugar dos sintomas.

Na década seguinte, Beata Bakker-de Pree $(1984,1987 a)$ contrastou o modelo de Goldiamond com a abordagem vigente na terapia comportamental clássica, que focalizava em primeiro lugar extinguir respostas problemáticas. A autora reagiu contra o raciocínio mecanicista inerente na abordagem neo-pavloviana, que tinha desenvolvido uma tecnologia para eliminação de sintomas e de comportamentos inadequados. A alternativa que Bakker-de Pree propôs foi criar espaço para que o cliente desenvolvesse repertórios bem-sucedidos, reconstruindo seu próprio estilo de lidar com os desafios e as oportunidades de sua vida. O termo neerlandês construcioneel, que ela usa para indicar essa visão, e que foi traduzido numa publicação anterior como construcional (Vandenberghe, 2003), é referido no presente artigo com o termo construtivo.

De acordo com a autora (Bakker-de Pree, 1985, 1987a), sintomas neuróticos emergem quando as estratégias habituais de esquiva ativa da pessoa não têm mais efeito. Esquiva ativa é comportamento expresso, ou seja, a pessoa age, evitando assim eventos aversivos. Difere da esquiva passiva, em que a pessoa não age e, pela sua passividade, evita conseqüências danosas ou desagradáveis. Cada um de nós possui suas próprias estratégias típicas de esquiva ativa que marcam sua personalidade. Trata-se de estratégias que nos protegem de males e geram, como efeitos colaterais, sentimentos de segurança e força.

O terapeuta faz uma análise dos setores saudáveis, bem-sucedidos, do comportamento do cliente, para determinar quais partes da construção total precisam de suporte. Os sentimentos são efeitos colaterais das contingências e podem servir como dicas para avaliar os comportamentos. De acordo com Bakker-de Pree (1984, 1987a, 1987b), uma pessoa é particularmente vulnerável a problemas psicológicos quando sua regulação comportamental é dominada em excesso por esquiva ativa, apresentando uma escassez de outros comportamentos como fuga, esquiva passiva, aproximação etc.

A pessoa fica ainda mais vulnerável quando grande parte do seu repertório de esquiva ativa é especializada em protegê-la contra a eventualidade de uma vivência aversiva, que pode ser descrita como invalidação pessoal ou desqualificação. Na sua teorização, a autora atribui grande importância às estratégias que as pessoas usam para evitar essa invalidação. No cotidiano, uma pessoa investe muito esforço em evitá- 
la: oferece ajuda para não ser insensível, desenvolve uma rotina meticulosa para não ser incompetente, gasta para não ser pão-duro, conversa para não ser antipática, se esconde para não ser extravagante. Evitar sinais de invalidação afeta o bem-estar. Em termos leigos, talvez seja isto a auto-estima.

Sintomas psicológicos só emergem quando as estratégias de esquiva ativa não funcionam mais. A pessoa não consegue mais evitar a vivência de invalidação. Isto pode acontecer em virtude de uma mudança situacional ou de uma passagem para uma nova fase de vida. Certos aspectos situacionais indicaram, num emprego anterior, que um determinado comportamento de esquiva ativa pode funcionar; mas, num novo ambiente de trabalho, é possível que estes não sejam mais relacionados com a probabilidade de sucesso. Esses aspectos deixam, assim, de funcionar como estímulos discriminativos. Passando da fase de adolescência para a idade adulta ou de um papel social para outro, o mesmo problema pode ocorrer. Para a pessoa que não dispõe de estratégias alternativas, o ambiente pode tornar-se ameaçador, e respostas encobertas alarmantes podem emergir. Nessa situação, a pessoa geralmente se volta contra o que sente dentro de si, tentando modificar seus sentimentos e pensamentos. Essa atitude leva necessariamente à derrota, porque os eventos encobertos são nada mais do que sinais de que algo está errado na sua relação com seu ambiente. Oportunidades reais para mudar o ambiente e entrar novamente em contato com sinais de segurança passam despercebidas enquanto a pessoa se concentra no seu mundo interior, usando todos os seus recursos na luta contra o que sente dentro de si (Bakker-de Pree, 1984).

O terapeuta não segue o cliente nas tentativas de mudar seus pensamentos negativos e sentimentos aversivos. Ao contrário, procura saber quais maneiras de lidar com o ambiente externo podem dar certo. Faz perguntas sobre ocorrências do cotidiano, nas quais a pessoa estava livre dos sintomas. Quando o cliente quer falar sobre os seus problemas, o terapeuta escuta-os com atenção, respeitando esse instante até que o cliente se mostre realmente pronto para falar sobre momentos em que estava livre de ansiedade ou depressão. Depois de identificar uma situação não problemática, os comportamentos e sentimentos são esmiuçados à procura dos estímulos discriminativos que estavam presentes nessa situação e controlavam o comportamento bem-sucedido do cliente. Assim, o cliente aprende a identificar estímulos que oferecem oportunidades para a ação produtiva no seu dia-a-dia (Bakker-de Pree, 1987a).

Uma técnica importante nessa fase é a pergunta de contraste: o terapeuta contrasta o que aconteceu na situação que está sendo analisada com o que poderia ter acontecido se o cliente não tivesse emitido o comportamento bem-sucedido. As respostas podem conter dicas sobre as contingências relevantes às quais o cliente estava reagindo, e, dessa 
forma, sobre a função (por exemplo, fuga, esquiva ou aproximação) do comportamento em questão (Bakker-de Pree, 1987a, 1987b).

O questionamento começa com perguntas como:"Você pode mencionar alguma ação que te fez bem?",e continua com:"O que poderia ter acontecido se você não tivesse agido dessa forma?". Quando fica claro que o comportamento bem-sucedido é alguma forma de esquiva ativa, outra pergunta pode ser feita: "Que tipo de pessoa você seria se não tivesse agido dessa forma?". Respostas como "Eu teria sido uma pessoa irresponsável" ou "Os outros teriam achado que sou um babaca" indicam que o cliente estava evitando desqualificação ou invalidação social. Nesse caso, o terapeuta pode prosseguir com perguntas para identificar conseqüências do comportamento bem-sucedido que serviam como sinal de segurança para o cliente. Respostas como "Foi o sorriso na cara dela" ou "Ninguém achou ruim" podem apontar para sinais de segurança que são importantes para o cliente. Enfim, depois de ter verificado que um episódio relatado realmente representava um padrão de esquiva que é funcional para o cliente (em outras palavras, um padrão que pode fazer parte do seu estilo comportamental), o terapeuta prossegue com outras perguntas, buscando identificar o estímulo discriminativo ao qual o cliente reagiu quando emitiu o comportamento (Bakker-de Pree, 1987a, 1987b).

A partir dessas informações, elabora-se um modelo que descreve o padrão de regulação comportamental predominante no repertório geral do cliente. Esse modelo especifica quatro tipos de informação: (1) as classes de estímulos discriminativos mais relevantes e (2) as classes de respostas que levam (3) aos sinais de segurança que constituem o contraste com (4) os sinais de invalidação que o cliente consegue evitar. $\mathrm{O}$ modelo deve ser testado repetidamente durante a terapia, a partir de predições derivadas dele. Em geral, a predição é que sintomas vão aparecer quando os estímulos discriminativos relevantes para os repertórios predominantes do cliente estiverem ausentes, ou quando, na presença dos estímulos discriminativos, o cliente for impedido de usar suas estratégias habituais (Bakker-de Pree, 1987b).

Em geral, o cliente retoma sua vida de maneira satisfatória após entrar novamente em contato com contingências relevantes que controlam suas estratégias mais bem-sucedidas. Os padrões de esquiva ativa que caracterizam seu estilo de personalidade podem vir a dominar outra vez suas interações com o ambiente. Retomar a maneira idiossincrática em que ele aprendeu a interagir com o meio permite-lhe enfrentar de forma saudável sua vida, sem precisar de sintomas. Porém, se a diversidade dos seus repertórios é limitada, ele continua sendo vulnerável, visto que podem surgir novas situações em que essas estratégias não sejam funcionais (Vlamings, 1999). 
Num tratamento estendido, o terapeuta busca prevenir a recaída. Procura promover outras classes de comportamento que podem estar fracas ou ausentes no repertório do cliente, como comportamentos de fuga e de aproximação. Nessa fase da terapia, o cliente aprende a lidar de maneiras variadas com as situações do seu cotidiano, para que dependa menos das suas estratégias de esquiva ativa. Uma diversificação maior das táticas de vida pode levar a uma diminuição da importância de sinais de segurança e aumentar o valor de outras classes de reforçadores para o cliente.

No trabalho de diferenciação livre (Bakker-de Pree, 1983), o terapeuta pede ao cliente para imaginar-se numa situação recente em que, confrontado com um estímulo aversivo ou um estímulo positivo, não tenha reagido com comportamento de fuga ou de aproximação. É importante que a ausência da fuga do estímulo aversivo, ou a falta de aproximação do estímulo positivo, se deva à predominância da esquiva de um possível sinal de invalidação. Deve-se tratar de uma situação em que o cliente não reagiu e, por isso, deixou de eliminar algo ruim ou deixou de aproveitar algo de bom. Ele perdeu chances porque, naquele momento, estava sob controle de uma ameaça, tentando evitar uma desqualificação pessoal.

O terapeuta indaga o que o cliente imagina que teria acontecido se ele tivesse reagido ao estímulo desconsiderado aversivo ou positivo. As respostas do cliente são usadas para criar roteiros imaginários em que o cliente não se esquiva da eventual invalidação (ou seja, não busca obter os sinais de segurança), mas responde a outras oportunidades de obter reforço positivo ou negativo. Esses roteiros podem ser ensaiados repetidamente em dramatizações durante a sessão. Cada vez que, nesses exercícios, o cliente experimenta reagir às classes de estímulos que ele tinha até então negligenciado, o terapeuta o confronta com a conseqüência temida, a saber, o sinal de desqualificação pessoal. Nesse treino, o cliente aprende a enfocar diversos estímulos e tolerar o desconforto advindo dos sinais de invalidação. A importância de evitar esses sinais se torna menos intensa do que a de fugir de prejuízos e aproveitar oportunidades (Bakker-de Pree, 1983, 1987a).

É preciso ressaltar que o cliente não recebe instruções sobre as maneiras pelas quais deveria reagir aos estímulos. É a criatividade dele que determina o conteúdo dos ensaios. Do ponto de vista do terapeuta, 
o reforçamento de novos comportamentos acontece de forma completamente cega, porque as preferências do clínico, concernindo o que o cliente deveria fazer numa certa situação, são consideradas irrelevantes (Beenackers, Bruins, \& Prins, 1996). Não é na terapia que as novas estratégias se modelam, mas no contato com as contingências naturais no cotidiano do cliente (Vlamings, 2001). Na sessão, o cliente apenas aprende a desconsiderar as ameaças da desqualificação, para poder entrar em contato com contingências que ofereçam oportunidades diferentes.

O cliente não deve adquirir comportamentos propostos pelo terapeuta, mas se tornar mais atento a possibilidades de agir à sua maneira, sem se esquivar de ameaças imaginárias. Também não é a intenção eliminar o comportamento de esquiva ativa, nem o impacto dos sinais de segurança, mas sim aumentar a influência de uma variedade de estímulos concretos capazes de controlar comportamentos de fuga e aproximação que tornam a vida mais rica. O efeito perseguido pela terapia é uma mudança na importância relativa dos estímulos do cotidiano. $\mathrm{O}$ cliente começa a fazer mais o que lhe faz bem e pára de fazer o que não vale a pena. Mudando as táticas de vida, a pessoa não só aproveita meIhor as oportunidades existentes, como ainda cria novas oportunidades. São as conseqüências dos novos comportamentos que vão selecionar e moldar os repertórios do cliente.

Ponto central na abordagem de Bakker-de Pree (1987a) é que o terapeuta não sugere topografias comportamentais adequadas (como, por exemplo, assertividade) para o cliente, mas atua para ajudá-lo a retomar ou aperfeiçoar suas próprias estratégias - que o possibilitam agir com segurança e se sentir satisfeito com seu desempenho. $O$ terapeuta não se deixa levar pelo dualismo racional/irracional ou adaptado/inadequado: concentra-se num questionamento contínuo das funções dos comportamentos e dos estímulos do ponto de vista do cliente.

Em resumo, o tratamento consiste na retomada de estratégias que o cliente abandonou e na expansão do repertório operante através de um treino da habilidade do cliente em identificar estímulos mais relevantes do que os sinais de segurança. É o próprio cliente que, livremente, desenvolve novas estratégias para reagir a esses estímulos. $O$ terapeuta somente lança os desafios e pede ao cliente para avaliar seu desempenho, de acordo com os sentimentos que este lhe provocou. $O$ profissional não sugere soluções, nem avalia comportamentos, porque somente o cliente tem condições de decidir o que é bom para si mesmo.

Caracterização e comparação com abordagens contemporâneas

A abordagem terapêutica que discutimos neste artigo compartiIha com a psicoterapia analítica funcional de Kohlenberg e Tsai (1991/ 2001) um estilo discursivo de fazer-se a análise funcional da vida do 
cotidiano, e uma dedicação à observação minuciosa de como o cliente reage durante as sessões. Bakker-de Pree $(1987 a, 1990)$ afirma que o funcionamento típico do cliente também se revela durante a sessão na interação com o terapeuta. Tomamos o exemplo em que o modelo de funcionamento geral que o terapeuta construiu para certa pessoa mostra que ela se desempenha excessivamente para evitar ser tachada de irresponsável. Evitando essa ameaça simbólica, ela se coloca em situações aversivas para as quais não dispõe de estratégias eficazes de fuga. Nesse caso, o terapeuta poderá entender por que, na relação terapêutica, o cliente aceitou desafios com os quais não consegue lidar.

Na terapia ocorre uma aprendizagem em dois níveis, e ambos precisam ser cuidadosamente monitorados (Bakker-de Pree, 1987a). O primeiro é o do trabalho técnico que foi descrito acima. O segundo é o da relação terapêutica. Diferentemente do ponto de vista que prevalece na análise clínica do comportamento norte-americana (Dougher \& Hayes, 2000; Kohlenberg et al., 1993; Jacobson \& Christensen, 1996), a autora afirma que, na relação terapêutica, o cliente deve aprender habilidades que facilitem o trabalho técnico. $O$ terapeuta deve ser o mais objetivo possível. A pessoa do terapeuta, como também a do supervisor clínico, com o estilo particular que predomina no seu repertório, é inevitavelmente uma variável independente no processo (Bakker-de Pree, 1990); porém, a autora não considera a intimidade do relacionamento pessoal entre terapeuta e cliente como um instrumento de tratamento em si.

A opção tomada por Bakker-de Pree (1987a) difere, assim, da posição de Kohlenberg e Tsai (1991/2001), os quais propõem que a relação terapêutica é o instrumento ideal para promover vivências curativas. No entanto, a maneira em que as táticas predominantes do cliente determinam o relacionamento com o terapeuta não passou despercebida na análise da autora. Ela aponta que a relação terapêutica é tipicamente um contexto em que o cliente evita, de maneira ativa, qualquer forma de desqualificação pelo terapeuta. Assim, os padrões problemáticos ocorrem nesse relacionamento. $O$ cliente pode, por exemplo, não reagir a um estímulo positivo por estar se esforçando para evitar ser mal avaliado pelo terapeuta. Também as melhoras no repertório do cliente podem ser observadas nesse relacionamento. Isto fica claro quando a autora aponta que, ao mesmo tempo em que melhora o funcionamento do cliente, muda também a maneira de o terapeuta se relacionar com ele. Mesmo assim, a autora não é a favor de usar o relacionamento como oportunidade de aprender a lidar de maneiras variadas com outra pessoa. O campo da experimentação deve se situar, de acordo com ela, na vida fora da sessão terapêutica. 
A terapia de aceitação e compromisso (Hayes et al., 1999) e a terapia comportamental construtiva são aparentadas no seu entendimento do cliente e do processo patológico. Ambas procuram levar o cliente a trocar a segurança da miséria que ele conhece pelo risco do desconhecido. As duas abordagens entendem que, enquanto seu estilo de esquiva funciona satisfatoriamente, ele não descobre novos caminhos. De acordo com as duas abordagens, o cliente procura a terapia quando as estratégias de esquiva com as quais lidou com a vida até o momento não funcionam mais. Ele quer fazer mais um pouco de malabarismo para solucionar a crise no nível emocional sem precisar agir sobre as situações concretas da sua vida.

A terapia de aceitação e compromisso coloca mais ênfase na necessidade de mudanças drásticas (Hayes et al., 1999). Essa abordagem procura agravar a crise para desfazer o equilíbrio maligno que o cliente construiu como solução para as inseguranças de sua vida. Coloca diretamente em questão as estratégias limitadoras em que o cliente tinha se enclausurado antes de começar a ter problemas. Bakker-de Pree (1987a) reconhece que o estilo de esquiva que o cliente desenvolveu tem um valor adaptativo para ele. Porém, procura reforçar o edifício de repertórios de maneira a permitir soluções mais flexíveis e funcionalmente mais variadas.

Ambas as abordagens recusam o pedido do cliente de ajudá-lo a controlar sua ansiedade ou seus sentimentos de depressão. Tentar manipular o que sentimos é como tentar evitar que a água escorra da palma da nossa mão fechando-a e infligindo muita força com o punho. Apoiando-se no behaviorismo radical, sugerem que o esforço deve ser direcionado para fora. As oportunidades de mudança estão nas contingências, não no mundo debaixo da pele. Porém, enquanto Hayes et al. (1999) dedicam bastante tempo e energia a desconstruir os contextos sociolingüísticos que prendem o cliente na luta com seu sofrimento, Bakker-de Pree (1987b) começa desde o início com sua análise funcional do comportamento bem-sucedido.

Implícito no trabalho da autora, existe um paralelo com as idéias dos seus colegas norte-americanos, como Linehan (1993), Hayes (1987) ou Hayes et al. (1999) que escreveram a respeito do compromisso com a ação valorizada. Todos esses autores defendem a opção de aprender a tolerar pensamentos e sentimentos problemáticos, exatamente para poder concentrar-se em mudar sua relação com seu ambiente. Embora a autora não elabore essa filosofia explicitamente, o tratamento descrito por ela promove um estilo de vida em que a pessoa faz o que valoriza em vez de se trancar em padrões defensivos "dentro da sua cabeça". 
Pretendemos concluir que a terapia comportamental construtiva tem uma visão similar o suficiente com as terapias comportamentais mais divulgadas para poder iniciar um diálogo. Por outro lado, apresenta ênfases suficientemente inovadoras para fazer uma contribuição valiosa. $A$ abordagem não só oferece diretrizes técnicas originais, mas também uma filosofia do tratamento baseada em um compromisso com a individualidade e a autonomia do cliente. Embora seja fundamental para a filosofia da terapia comportamental, essa opção tem ficado, não raro, muito implícita nos modelos teóricos aos quais estamos acostumados.

\section{Constructional behavior therapy: Another side to behavioral treatment.}

Abstract: The present article discusses constructional behavior therapy, as developed by Beata Bakker-de Pree in the Netherlands. Its general philosophy of treatment is compatible with several of the wider known behavior therapies. But its original contribution consists in the explicit description of a number of important ingredients in clinical work that are not emphasized in the other behavioral models. These are the technique of free differentiation and the functional analysis of the client's healthy behavior.

Keywords: Behavior therapy. Behaviorism. Treatment model.

\section{A thérapie comportementale constructionelle ${ }^{1}$ : une autre face de la clinique comportementale.}

Résumé: Le present article offre une revue de la thérapie comportementale constructionelle' ${ }^{2}$, d'après sa formulation par Beata Bakker-de Pree, aux Pays-Bas. Sa philosophie générale de traitement est compatible avec celle des traitements comportementaux plus connus, mais sa contribution originelle réside dans la description explicite de quelques ingrédients importants du travail thérapeutique qui ne sont pas mis en lumière par d'autres modèles comportementaux. Il s'agit de la technique de la différenciation livre et de l'analyse fonctionelle des aspects sains du comportement du client.

Mots-clés: Thérapie comportementale. Béhaviorisme. Modèle clinique.

\section{Psicoterapia comportamental constructiva: una otra faceta de la clínica conductista.}

Resumen: En el presente artículo se describe la psicoterapia comportamental constructiva como elaborada por Beata Bakker-de Pree. La pers-

1 Não encontramos referências a thérapie constructionelle, mas sim a thérapie constructive. Em todo caso, como pode ser que se trate de coisas distintas e de uma proposta do autor, apenas registramos o achado.

2 Ver nota acima. 
pectiva, que se desarrolló en los Países Bajos, nos trae una filosofía del proceso terapéutico que es compatible con varias otras psicoterapias conductistas más conocidas. Incorpora una perspectiva valiosa en la descripción explícita de algunos componentes importantes del trabajo clínico, lo que la hace diferente de otros modelos conductuales, que no los destacan:la técnica de diferenciación libre y el análisis funcional del comportamiento saludable del cliente.

Palabras-clave:Terapia comportamental. Conductismo. Modelo clínico.

\section{Referências}

Bakker-de Pree, B. J. (1983). Vrije differentiatie: Een nieuwe gedragstherapeutische methode. Gedragstherapie, 16, 255-269.

Bakker-de Pree, B. J. (1984). De rijkdom van de leertheorie in de constructionele gedragstherapie. Gedragstherapie, 17, 179-197.

Bakker-de Pree, B. J. (1985). Het proces van de functionele analyse. In J.W. G. Orlemands, P. Eelen \& W. P. Haaijman (Orgs.), Handboek Gedragstherapie, A.15.3. (pp. 1-25). Deventer:Van Loghum Slaterus.

Bakker-de Pree, B. J. (1987a). Constructionele gedragstherapie. Nijmegen: Dekker en Van de Vegt.

Bakker-de Pree, B. J.(1987b). Behavior analysis of successful behavior:The device for identifying which stimuli, if wanting, cause neurotic disorder. Trabalho apresentado no XIII Encontro Anual da Association for Behavior Analysis. Nashville.

Bakker-de Pree, B. J. (1990). (On)opleidbaarheid van psychotherapeuten: De persoon van de opleider als onafhankelijke variabele. Tijdschrift voor Psychotherapie, 16, 258-265.

Beck, T., A., Rush, A., J., Shaw, B., F., \& Emery, G. (1979). Cognitive therapy of depression. New York: Plenum.

Beenackers, A. A.J.M., Bruins, B.E. E., \& Prins, F.S. (1996). Blinde versterking: Klachten verhelpen zonder erop in te gaan. MGV, 6, 607-618.

Coons, W. H. (1972). Psychotherapy and verbal conditioning in behavior modification. Canadian Psychologist, 13, 3-29.

Craske,M.G., \& Rowe, M.K.(1997). A comparison of behavioral and cognitive treatments of phobias. In G. C. L. Davey (Org.), Phobias. A handbook of theory, research and treatment (pp. 247-280). London:Wiley.

Dougher, M. J., \& Hayes, S. C. (2000). Clinical behavior analysis. In M. J. Dougher (Org.), Clinical behavior analysis (pp. 11-25). Reno: Context Press. 
Ellis, A., \& Grieger, R. (1977). Handbook of rational-emotive therapy. New York: Springer.

Eysenck, H.J. (1952). The effects of psychotherapy: An evaluation. Journal of Consulting Psychology, 16, 319-324.

Eysenck, H. J. (1959). Learning theory and behaviour therapy. Journal of Mental Science, 105, 61-75.

Eysenck, H. J. (1987). Behavior therapy. In H. J. Eysenck \& I. Martin (Orgs.), Theoretical Foundations of Behavior Therapy (pp. 3-36). New York: Plenum.

Goldiamond, L. (1974). Toward a constructional approach to social problems. Behaviorism, 2, 1-85.

Hayes, S. C. (1987). A contextual approach to therapeutic change. In N. S. Jacobson (Org.), Psychotherapists in clinical practice (pp. 327-387). New York. The Guilford Press.

Hayes, S. C., Strosahl, K. D., \& Wilson, K. G. (1999). Acceptance and commitment therapy: An experiential approach to behavior change. New York: Guilford.

Jacobson, N. (1997). Can contextualism help? Behavior Therapy, 28, 435443.

Jacobson, N. S., \& Christensen, A. (1996). Integrated couple therapy: Promoting acceptance and change. New York: Norton.

Kohlenberg, R. J., Bolling, M. Y., Kanter, J. W., \& Parker, C. R. (2002). Clinical behavior analysis:Where it went wrong, how it was made good again, and why its future is so bright. Behavior Analyst Today, 3, 248-253.

Kohlenberg, R. J., \& Tsai, M. (2001). Psicoterapia analítica funcional: criando relações intensas e curativas. Santo André, SP: ESETec. (Trabalho original publicado em 1991)

Kohlenberg, R. J.,Tsai, M., \& Dougher, M. (1993). The dimensions of clinical behavior analysis. The Behavior Analyst, 16, 271-282.

Krasner, L. (1962). The therapist as a social reinforcement machine. In $\mathrm{H}$. H. Strupp \& L. Luborsky (Orgs.), Research in psychotherapy (Vol 2, pp. 61-94). Washington: American Psychological Association.

Linehan, M. M. (1993). Cognitive-behavioral treatment of borderline personality disorder. New York: Guilford Press.

Neimeyer, R. A., \& Mahoney, M. J. (1997). Construtivismo em psicoterapia. Porto Alegre: Artes Médicas. (Trabalho original publicado em 1995).

Rachman, S. (1998). Anxiety. Hove:Taylor and Francis. 
Stuart, R. (1969). Operant-interpersonal treatment for marital discord. Journal of Consulting and Clinical Psychology, 36, 675-682.

Vandenberghe, L. (2003). Terapia comportamental construcional do borderline. In M. Z. Brandão, F. C. Conte, F. S. Brandão, Y. K. Ingberman, C. B. Moura, V. M. Silva \& S. M. Oliane (Orgs.), Sobre comportamento e cognição Vol. 12: Clínica, pesquisa e aplicação (pp. 92-96). Santo André: ESETec.

Vlamings, H. S. M. (1999). Basic treatment in constructional behavior therapy: Restoring and stregthening present patterns of behavior regulation. In B. E. E. Bruins, J. D. van Delden-van der Wolf, E. P. Van der Meijde, F. S. Prins, H. S. M. Vlamings \& P. J. H. Andreoli (Orgs.). Constructional behavior therapy: A novel development in the Netherlands (pp. 18-22). Utrecht: Stichting Constructionele Gedragstherapie.

Vlamings, H. S. M. (2001). Restoring and strengthening present patterns of behavior regulation. In E. P. Van der Meijde (Org.), Let natural reinforcers do their job. Simpósio apresentado no I Encontro Internacional da Association for Behavior Analysis. Veneza.

Wolpe, J. (1976). Behavior therapy and its malcontents: Multimodel eclecticism, cognitive exclusionism and exposure empiricism. Journal of Behavior Therapy and Experimental Psychiatry, 7, 109-116.

Recebido em: 21/06/2006

Aceito em: 05/02/2007 